\title{
La pintura cerámica valenciana y sus sistemas de reintegración a través de la metodología documental, gráfica y escrita
}

\author{
Ignasi Gironés Sarrió, Vicente Guerola Blay
}

\begin{abstract}
Resumen: Con nuestro estudio tratamos de aportar una solución a la siempre comprometida disciplina de reintegración formal, cromática y verbal en materia de azulejería devocional. La gran cantidad de circunstancias que encierra el hecho creativo, mecenazgo, iconografía, fuentes, formato, etc., hacen necesaria una aproximación al hecho creativo de la obra desde su propia fenomenología. En este sentido, con nuestro estudio tratamos de protocolizar una pauta de trabajo alrededor de la revisión de las fuentes gráficas utilizadas por prácticamente todas las manufacturas valencianas a lo largo del siglo XVIII. Así, el conocimiento de las fuentes, directas o indirectas, precisa de un pormenorizado trabajo de investigación para localizar depósitos, archivos y colecciones donde poder sistematizar o incluso protocolizar un programa de adquisición de información.
\end{abstract}

Palabras clave: reintegración de azulejería, fuentes gráficas, azulejería valenciana, fuentes documentales, recursos semánticos, iconografía de la Trinidad, Ontinyent

\section{Valencian ceramic tilework and its reintegration systems based on using documentary, graphic and written methodology}

\begin{abstract}
With this work we treat to contribute a solution to the always committed discipline of formal reintegration, chromatic and verbal about devotional ceramic tilework. The circumstances that shuts the creative fact, patronage, iconography, sources, format, etc. do necessary an approximation to the creative fact of the work from his own phenomenology. In this sense, with our study we try to create a work guide around the review of the graphic sources used by practically all the Valencian factories throughout the XVIII century. Thus, the knowledge of the sources, direct or indirect, requires a detailed research work to locate deposits, archives and collections where an information acquisition program can be systematized or created.
\end{abstract}

Keywords: tilework reintegration, graphic sources, valencian tilework, documentary sources, semantic resources, iconography of the holy Trinity, Ontinyent

\section{A pintura cerâmica valenciana e os seus sistemas de reintegração através da metodologia documental, gráfica e escrita}

Resumo: Com o nosso estudo pretendemos contribuir com uma solução para a sempre comprometida disciplina de reintegração formal, cromática e verbal em matéria de azulejos devocionais. O grande número de circunstâncias que engloba o ato criativo, mecenato, iconografia, fontes, formato, etc., torna necessário abordar o ato criativo da obra a partir de sua própria fenomenologia. Nesse sentido, com o nosso estudo procuramos protocolar um padrão de trabalho em torno da revisão das fontes gráficas utilizadas por praticamente todas as manufaturas valencianas ao longo do século XVIII. Assim, o conhecimento das fontes, diretas ou indiretas, requer um trabalho minucioso de pesquisa para localizar depósitos, arquivos e acervos onde se possa sistematizar ou mesmo protocolar um programa de aquisição de informação.

Palavras-chave: reintegração de azulejos, fontes gráficas, azulejos valencianos, fontes documentais, recursos semânticos, iconografia da Trindade, Ontinyent 


\section{Introducción}

Puede que, en muchos casos, uno de los debates más controvertidos en el proceso de restauración de obras de arte se centre especialmente en la reintegración formal o cromática de sus partes faltantes o lagunas y, mucho más, cuando circunstancias como forma, extensión y ubicación comprometen seriamente la correcta lectura o apreciación de la obra. Es evidente que el término"perdida" conlleva, en cierto sentido una aseveración de "perjuicio", mientras que la reintegración suele llevar asociado el estigma de "prejuicio" (Guerola 2010). Respecto a esta cuestión no escapa la azulejería, si bien su propia caracterización, tanto técnica, simbólica, como estética marcan, en distintas ocasiones, la solución en orden a su proceso de reintegración.

La azulejería dentro de los márgenes de la tipología reconocida como "cerámica arquitectónica" o "de serie" sería el ejemplo menos problemático en el momento de abordar una posible recuperación de su trama figurativa u ornamental, así como del tratamiento de su policromía. El innegable hecho de tratarse de un tipo de fabricación seriada y de una decoración estandarizada, otorga a su posible reintegración de factores de innegable facilidad de interpretación o retoque. Principalmente, porque disponemos de un modelo a partir del cual llevar a término su proceso de retoque o reproductibilidad.

Aproximadamente hacia 1740 se inicia en Valencia una producción de azulejería basada en unas características singulares, de acento propio y autónomo. En el caso que nos ocupa las fuentes gráficas y estéticas se encuentran básicamente relacionadas, siguiendo un orden progresivo de importancia, empezando con la xilografía y el grabado, seguido de la imaginería, la pintura y el dibujo.
La estampa xilográfica, por su carácter popular e inmediato, es probablemente con diferencia la fórmula de mayor influencia en las fuentes de inspiración de la azulejería devocional. La imaginería, la pintura y los dibujos fueron a menudo fuente de inspiración, si bien se pueden documentar muy pocos casos de interpretación directa, dado que casi siempre solía mediar entre ellos una fuente gráfica.

Paralelamente también se toman en cuenta otros aspectos que pueden facilitar y aportar una comprensión gráfica de las obras basadas en el reaprovechamiento de dibujos preparatorios, la reutilización de estarcidos, o la copia literal y estandarizada de algunas imágenes devocionales, así como la existencia de registros fotográficos previos a una eventual pérdida de azulejos.

Solo la utilización de estos recursos puede dar validez al proceso de retoque de estas obras de azulejería, validez que se fundamenta en la recuperación de su mensaje primigenio y de su retórica visual, de otro modo se puede incurrir en trasgresiones al sentido simbólico e icónico de las obras. [Figura 1].

Centrándonos en el amplio abanico de posibilidades de sistemas de reintegración que parten, obligatoriamente de postulados teóricos y de las innegables especificidades que requiere cada tipo de intervención en particular, podemos abrir un amplio y diversificado espectro de modelos de reintegración en materia de azulejería. Bien es cierto que, en el terreno de la cerámica arquitectónica, prácticamente siempre seremos conocedores de repertorio gráficoornamental y de su espectro colorimétrico $y$, por tanto, sabremos de antemano cómo fueron los azulejos en su origen. Mientras que, en la pintura cerámica de paneles devocionales y costumbristas, de mayor complejidad formal
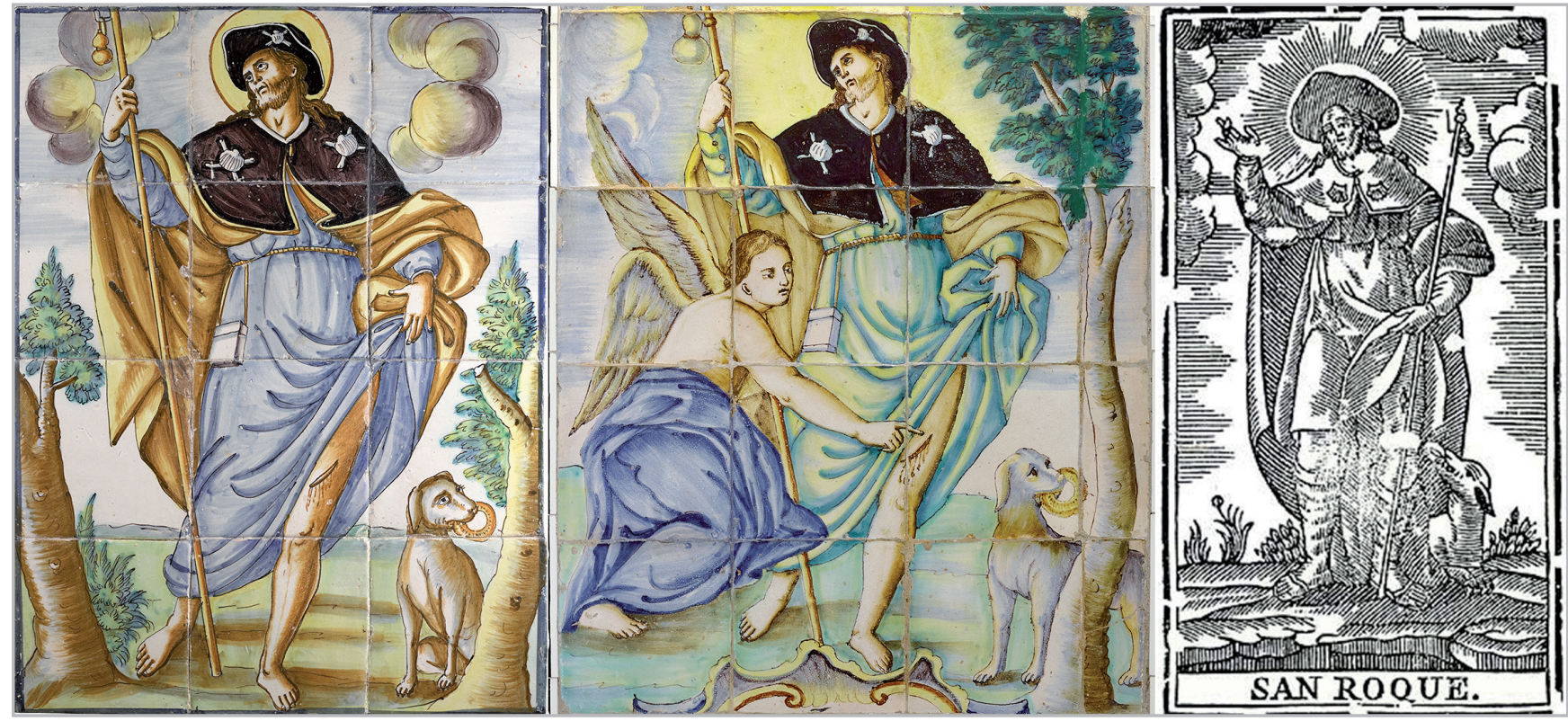

Figura 1.- Ejemplo de utilización de un mismo estarcido para la realización de diversas obras junto la fuente gráfica de inspiración. De izquierda a derecha: San Roque, panel cerámico perteneciente a la Colección "Fundación La Fontana"; San Roque, panel cerámico procedente de una colección particular de L'Alcúdia, y San Roque, xilografía del repertorio de la imprenta Laborda. 
y narrativa, deben rastrearse necesariamente sus fuentes gráficas para una correcta interpretación o propuesta de reintegración.

\section{La técnica de Ne rian faire}

Podríamos traducirlo, no solamente como la fórmula de "no hacer nada" sino, como la de hacer prevalecer la visión descarnada, alterada y con pérdidas del original por encima de cualquier acción de restauración. El término Ne rian faire aplicado a los sistemas de reintegración fue acuñado por primera vez por Ségolene Bergueon desde su responsabilidad al frente de los Museos Nacionales de Francia (1990). Esta técnica puede poner el énfasis en la transmisión de los valores de la obra como objeto de uso, en el caso que aparezcan desgastes o erosiones en la superficie esmaltada o del soporte; como también, en el valor otorgado a las piezas procedentes de hallazgos arqueológicos. Esta práctica sería especialmente indicada para azulejería seriada arquitectónica y menos recomendable para paneles devocionales o costumbristas por cuanto la trama figurativa requiere de ejercicios que respondan a las necesidades de la solución figurativa de las obras.

\section{La tinta neutra}

El ejercicio de este sistema de reintegración supone la recuperación de los fragmentos de soporte y esmalte perdidos a través de la restitución volumétrica o del estrato estannífero. Teóricamente persigue la "neutralización" de las partes faltantes a través de una tinta "sin timbre", que facilite y otorgue valor a las partes originales conservadas. En la práctica, no existe una sola tinta neutra capaz de responder de forma uniforme a este prototipo de reintegración. La tinta neutra se adaptará a los valores cromáticos del original de tal modo que no sea mayor, ni menor, en brillo y saturación a la suma de los colores presentes en la obra. La tinta neutra fue muy utilizada el pasado siglo como sistema en la restauración de pintura mural buscando, en la mayoría de los casos, operaciones poco invasivas y con connotaciones, podríamos denominar, de carácter arqueológico.

Se puede utilizar la tinta neutra a partir de la propia coloración del mortero con el que se rellenan las partes perdidas o a través de la aplicación de color sobre la base de relleno. En cualesquiera de las soluciones también se pueden ofrecer soluciones de texturización a partir de la granulometría del mortero o de la aplicación de una capa pictórica de reintegración a través de sistemas de pulverización.

\section{Reintegración por aproximación lineal o formal}

Este sistema supone un grado o escala a nivel de intervención, superior respecto de la tinta neutra, dado que parte de los principios de aquella con una solución gráfica superpuesta. Quiere esto decir que, a partir de una tinta neutra, se pueden conseguir tratamientos de reintegración de la imagen perdida a través de dibujos sinópticos de las partes faltantes. Esta sería la definición más ajustada al sistema: la inclusión, sobre una capa monócroma y sin timbre, de un dibujo esquemático que ayude al espectador a la comprensión de las partes faltantes con la mínima información gráfica.

La elección de este sistema puede llegar a provocar, en algunos casos, situaciones que propicien la propuesta de aproximación lineal de partes sobre las que existe poca información, si bien en algunos casos el conocimiento de fuentes gráficas puede ayudar a paliar estas carencias. Esta fórmula de reintegración puede ser igualmente utilizada en cerámica de serie o en paneles devocionales y costumbristas y constituirse en una solución intermedia entre posturas extremas de reintegración.

Cabe señalar que una desacertada reintegración gráfica con este sistema puede convertir el trabajo en una suerte de comic, o puede terminar, por exceso de información, en convertirse en un rival respecto de la obra, perdiendo cuanto de "respecto al original" debe cumplir un proceso de restauración en materia de retoque gráfico y formal (Gironés y Guerola 2014). [Figura 2]

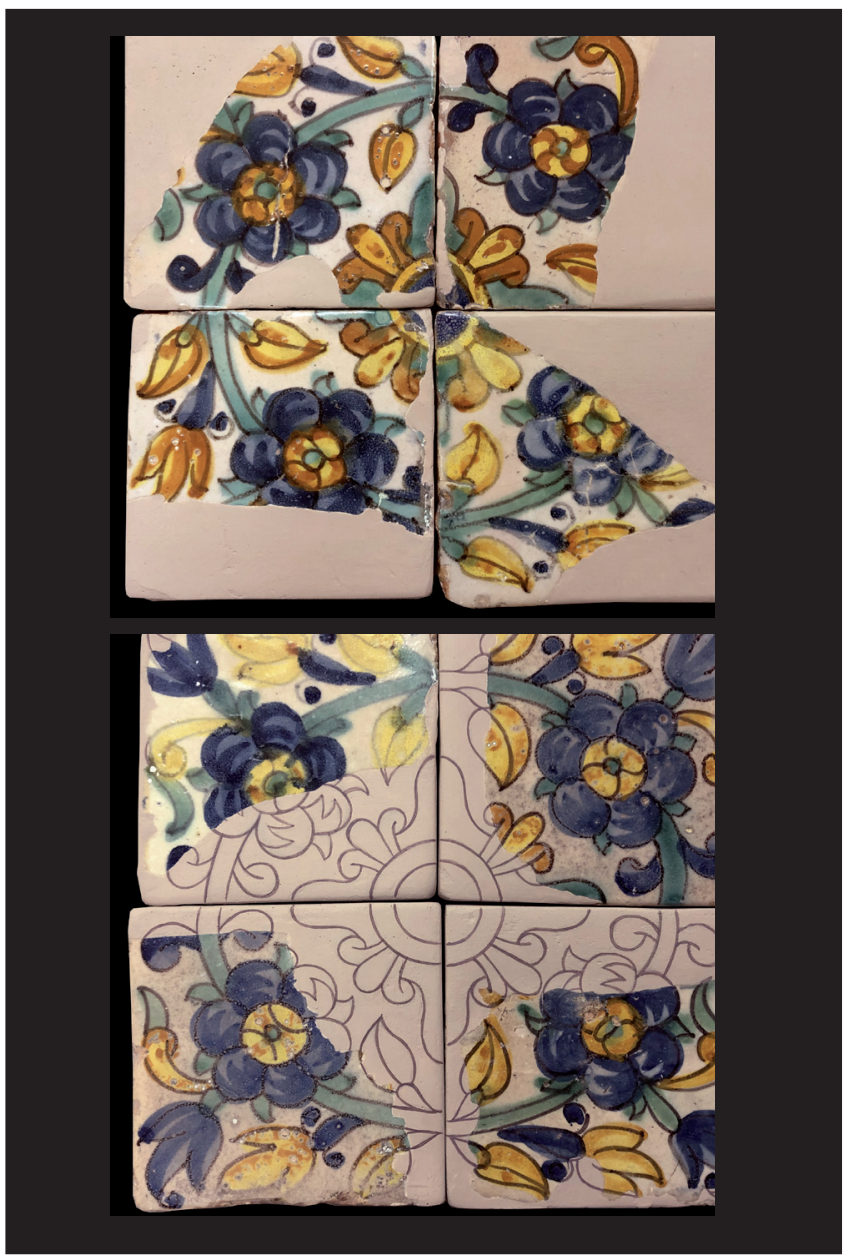

Figura 2.- Azulejos de cuarto ornato. Manufactura valenciana del primer tercio del siglo XVIII. Ejemplos de reintegración neutra y por aproximación lineal. 
Sistemas de reintegración de forma y croma. "La selección del color" y el "tratteggio"

Se trata del sistema mayoritariamente aceptado y el más utilizado para la reintegración puntual de pequeñas lagunas, donde la parte faltante puede ser recompuesta formal y cromáticamente. Los procedimientos básicos de reintegración en azulejería siguen muy de cerca aquellos comúnmente aceptados para el retoque de superficies pictóricas como la pintura de caballete, la mural o la escultura polícroma, a base de tratteggio vertical, modulado, entrecruzado, multidireccional, etc. También se utiliza el sistema de puntillismo para faltantes de pequeña extensión.

Metodológicamente estos sistemas parten de la denominada "reintegración por selección del color" que definiese en la segunda mitad del pasado siglo Umberto Baldini (1981) y Ornella Casazza (1981) en su Teoría de la Restauración desde el Opificio delle pietre dure de Florencia. Esta práctica persigue la reconstrucción de la trama formal y cromática de la zona perdida, a través de la lectura cromática de la zona circundante a la laguna para realizar un rayado por medio de colores puros sin alteraciones de claroscuro.

Desde los sistemas discernibles de retoque, por medio de una texturización óptica como el tratteggio y todas sus variantes, hasta los sistemas de reintegración no discernible, incluyendo los ex novos, existe un amplio repertorio de fórmulas metodológicas de actuación.

Aún a pesar de que algunos autores tratan de asimilar el término tratteggio a la escuela florentina y rigatino a la romana, ambas etimologías responden a un mismo concepto de reintegración, más allá de tratarse de pequeños trazos entrelazados o de un trazo continuo (Guerola 2008).

\section{El reglatino}

Este término acuñado y muy introducido en el ámbito de habla hispana es una variante del tratteggio según la fórmula del Instituto Centrale per il Restauro de Roma. Se trata de un rayado sutil, continuo y muy tupido semejante al tejido de un tapiz. Ya Cesare Brandi (2002) en sus discursos define en un primer momento este sistema como i filamenti, para pasar en escritos sucesivos a denominarlo tratteggio-rigatino. La escuela de restauración en Italia presenta dos técnicas de reintegración relativamente enfrentadas en su consistencia metodológica. De una parte, la florentina, con un sistema de texturiación óptica a base de pequeños trazos verticales superpuestos, mientras que la escuela romana, propugna líneas a través de un rayado vertical continuo que se ejecuta de extremo a extremo de la laguna.
La variante hispánica de este sistema, muy utilizada en pintura mural y azulejería, suele realizarse con lápices acuarelables, a diferencia del sistema italiano a pincel, y toma etimológicamente su nombre de "regla", por el instrumento con el que realizar de forma mecánica el trazado de las líneas. Generalmente se practica sobre una base de colores a bajo tono respecto de la laguna para a continuación llevar a término el rayado con la finalidad de ajustar la trama figurativa y cromática. [Figura 3].

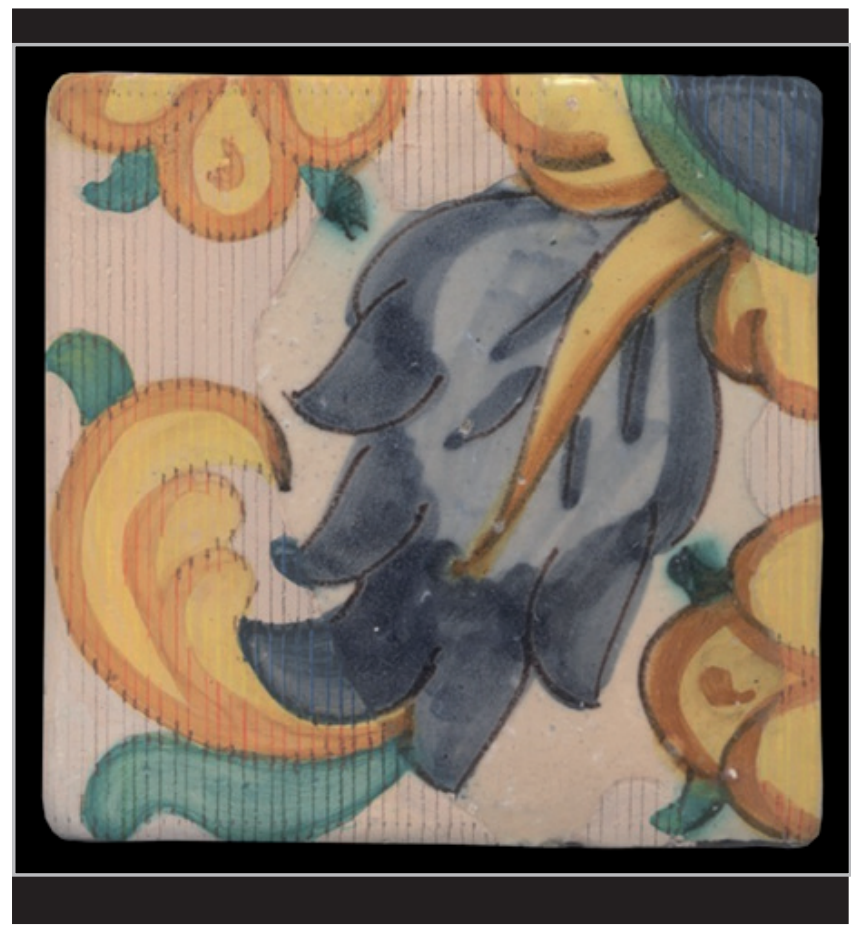

Figura 3.- Azulejo valenciano de cuarto ornato denominado "voladoret". Primer tercio del siglo XVIII. Ejemplo de reintegración por reglatino.

\section{La reintegración no discernible}

Suele practicarse en aquellos casos donde el tamaño de las lagunas no permite la utilización de sistemas de texturización óptica. También se utiliza ante imperativos derivados del uso y función patrimonial. Casos como obras que han sufrido algún tipo de accidente o daño y que cumplían un sentido doctrinal a través de mensajes escritos o emblemas iconográficos. Mención especial merecen, en este sentido, las obras de carácter devocional, así como los imperativos derivados del mercado del arte y las antigüedades que generalmente demandan y se posicionan ante la práctica de la reintegración por fórmulas de reconstrucción (Gironés y Guerola 2008).

Evidentemente la reintegración no discernible, como indica su nombre, persigue la total reabsorción de la parte perdida de la obra a través de un sistema de retoque formal y cromático que rivaliza en su estructura visual con el original. [Figura 4] 


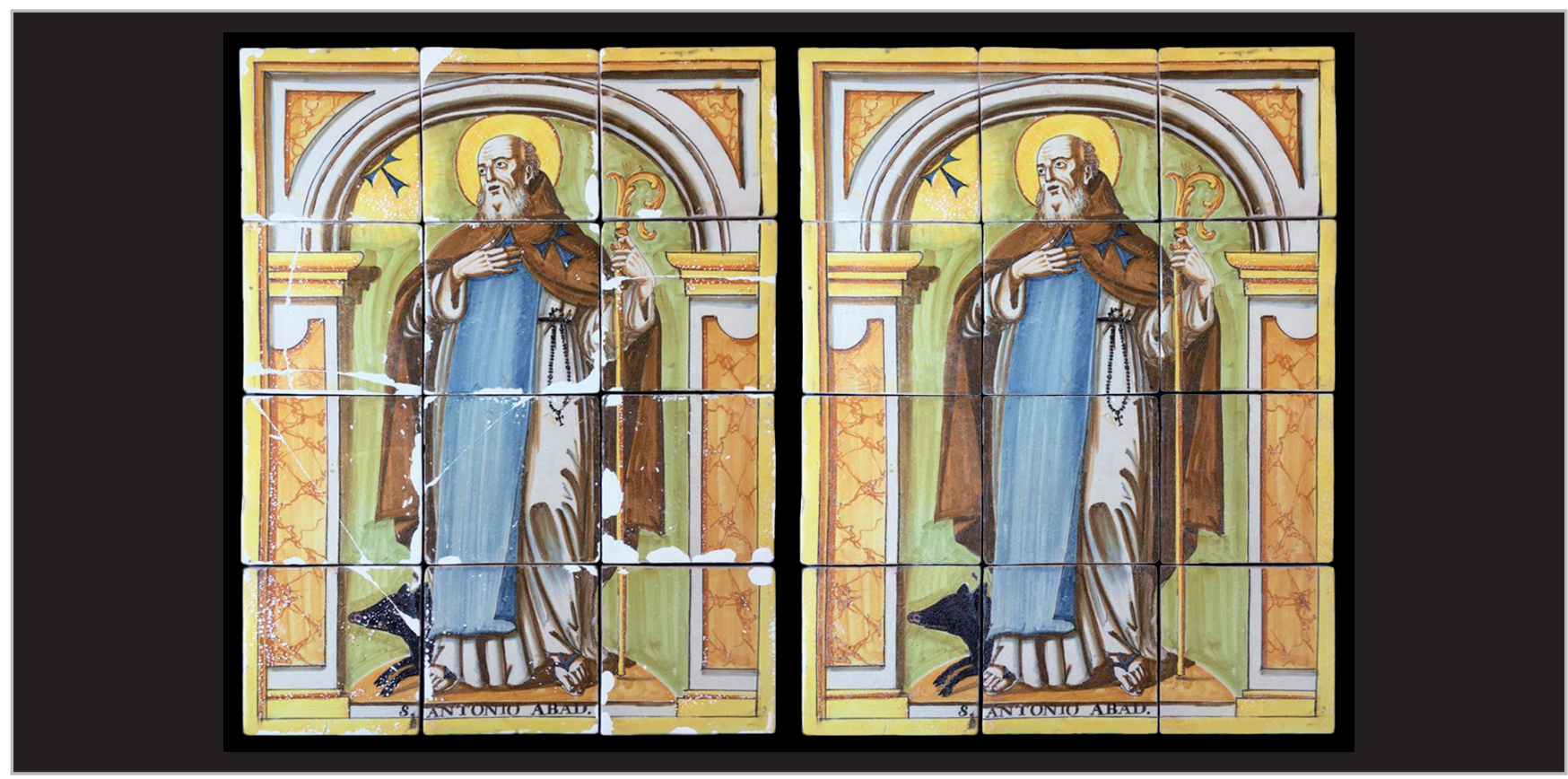

Figura 4.- San Antonio abad. Obrador valenciano. (Circa1780). Ejemplo de reintegración no discernible.

\section{Los ex novos}

Se trata, como su nombre indica, de piezas de azulejería de nueva factura interpuestas dentro del despiece de la obra original. Este sistema suele utilizarse bastante a menudo en cerámica arquitectónica seriada ante la ineludible necesidad de restitución de faltantes. La fórmula, a nivel ideológico, persigue la visión global del sentido primigenio del conjunto a través de la restitución de las piezas faltantes, además suelen generalmente grabarse los azulejos con inscripciones o sellos que manifiestan el origen ex novo de la reconstrucción. Esta señal permite documentar con facilidad las piezas incorporadas y restituidas en el proceso de restauración.

Ya hemos señalado la ventajosa opción que presenta este tipo de reintegración en materia de azulejería arquitectónica o de serie, en cambio, en azulejería narrativa devocional o costumbrista requiere de un estudio ciertamente pormenorizado de la conveniencia de su incorporación. En este sentido, es fundamental para llevar a término los ex novos tener un conocimiento de la fuente gráfica directa utilizada para la realización del panel. Rehuir de esta imperiosa necesidad documental puede llevar a errores de estrepitoso fracaso, así como a incongruencias simbólicas e icónicas a través de la incorporación de elementos figurativos ajenos a la obra.

Solo la utilización de estos recursos de investigación, en orden al estudio de las fuentes gráficas, puede dar validez al proceso de reintegración de estas obras de azulejería, aprobación que se fundamenta en la recuperación de su mensaje primigenio y de su retórica visual.

\section{La técnica del bajo nivel}

Más que de un sistema se trata de un proceso o técnica que puede ser interpuesto a muchas de las propuestas anteriores. Se trata de desnivelar en un estrato inferior la superficie de relleno o masillado provocando una disociación de superficies, de tal modo que el original prevalezca y se visualice en primer término, mientras que la zona reintegrada se encuentre en un estrato inferior, interpretándose como segundo término.

Está técnica puede ser aplicada, tanto en supuestos de reintegración neutra, como en sistemas de reintegración discernible y no discernible. En cualquier caso, puede ser una opción convencional para transformar cualquier sistema en una fórmula de diferenciación respecto del original a través de la denominada "reintegración a bajo nivel". [Figura 5]

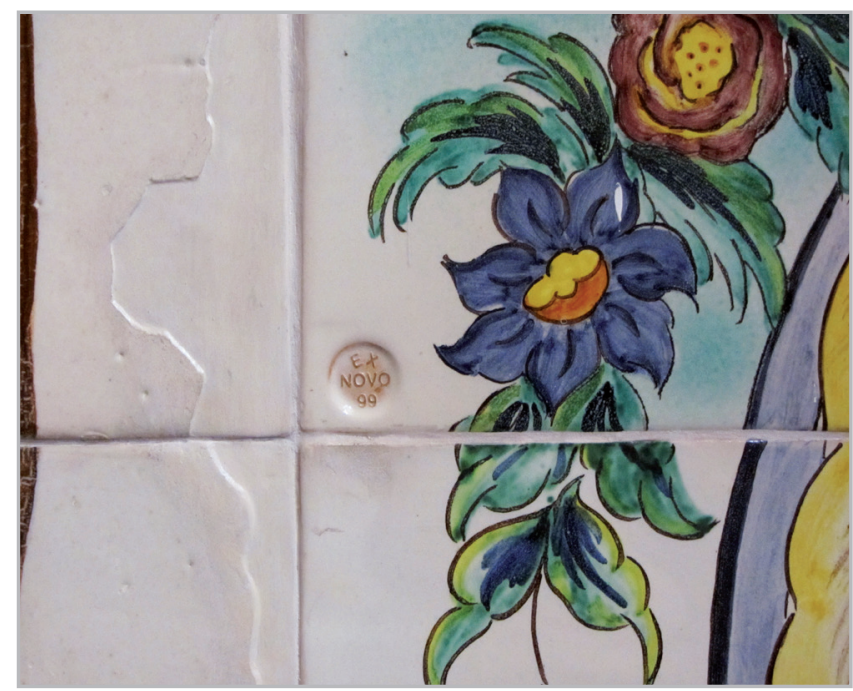

Figura 5.- Ejemplo de reintegración a bajo nivel y de ex novo. 
Caso de estudio: La Trinidad de Ontinyent. De la reintegración neutra en el original a la hipótesis virtual como sistema de apoyo museográfico.

La pintura cerámica devocional valencia, tal y como hemos podido comprobar en la narración precedente, sigue en algunos casos una serie de parámetros a través de los cuales se basa nuestra metodología para poder llevar a cabo una reintegración en aquellos casos más problemáticos (Guerola 2003; Gironés y Guerola 2016). En este sentido, nuestra experiencia se ha puesto en práctica para la reintegración de un panel cerámico cuya complejidad en su restauración gráfica y cromática suponía un esfuerzo práctico y conceptual. Para ello, ha sido fundamental el estudio y utilización de las fuentes gráficas de la obra, el conocimiento y análisis de las técnicas utilizadas en el obrador, y los estarcidos repetidos en la creación primigenia de este panel cerámico.

Laobraencuestión setrata deun panelcerámico, perteneciente a las producciones de pintura cerámica valenciana datado en 1790, que se recuperó en una excavación arqueológica en Ontinyent (La Vall d'Albaida - Valància) en el que aparece representado el tema de la Santísima Trinidad (Belda 2001). Está compuesto por un total de treintaicinco azulejos de 20,5 $x 20,5 \mathrm{~cm}$, con un despiece de cinco columnas y siete líneas y con unas dimensiones totales de 143,5 x 102,5 cm.

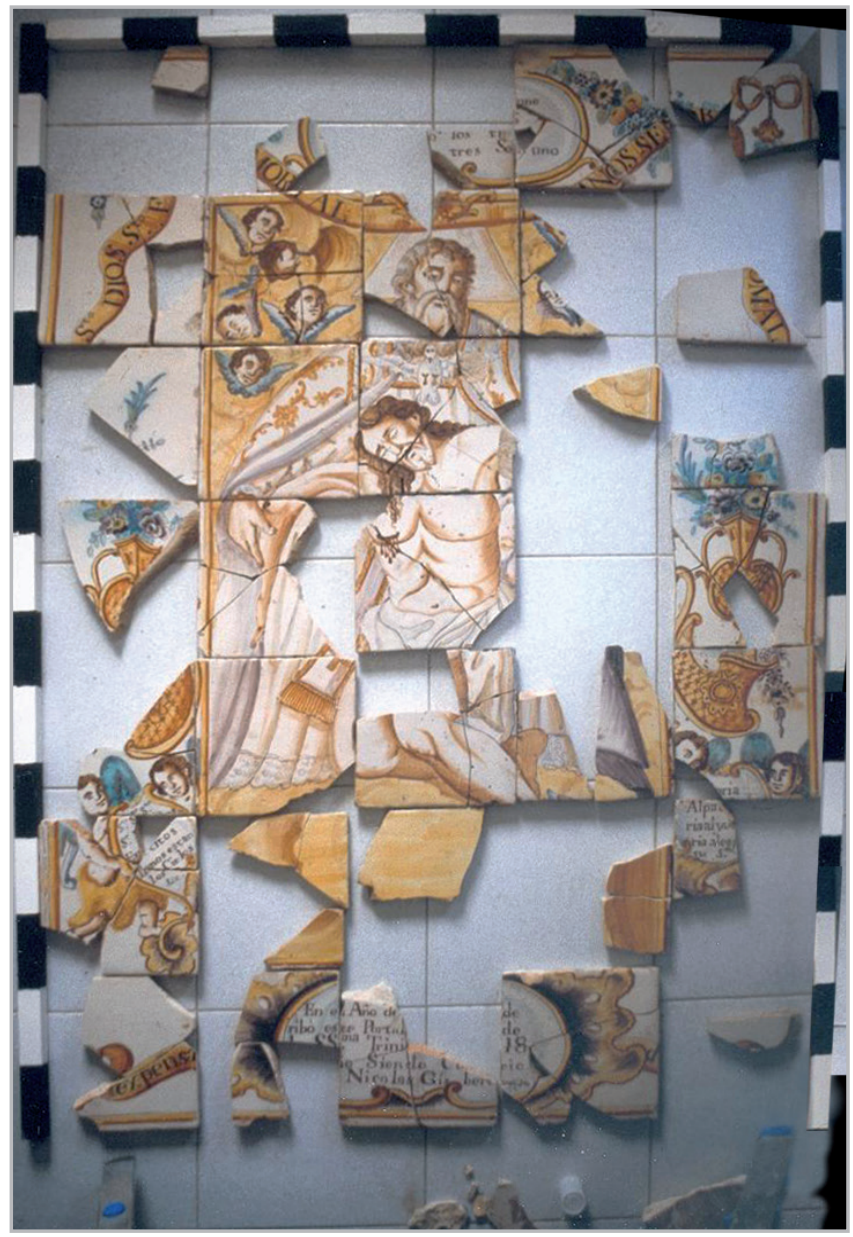

Figura 6.- Estado inicial de La Trinidad después del hallazgo en la excavación.
Su descubrimiento se produjo en dos campañas arqueológicas diferentes realizadas en la misma zona del barrio antiguo de Ontinyent, una en el año 1990 y otra en el 2000 (Ribera y Belda 2003; Ribera 2006), en la que en cada una de ellas se extrajeron un número determinado de fragmentos, aunque lamentablemente no se pudo recuperar la totalidad que conformarían el panel completo. Solamente se recuperó aproximadamente un $60 \%$ de la extensión del panel. No obstante, con la información de las partes recuperadas y con un proceso de documentación gráfica, histórica y documental se ha podido llevar a término una reintegración de tipo neutro en el original y una hipótesis de reconstrucción virtual, como ayuda a su presentación museográfica. [Figura 6].

La configuración de un total de 64 fragmentos fueron sometidos a los procesos básicos de restauración en este campo, en el que no nos vamos a detener por no ser el objeto del trabajo. Simplemente comentaremos que, tras su limpieza mecánica y desalación, éstos se encolaron. Posteriormente, se reconstruyeron las partes de pasta cerámica perdida en cada uno de los azulejos para que cada unidad pudiera, como mínimo, recuperar su volumetría original y permitir contemplar el conjunto en su dimensión primigenia. Para este proceso de restitución volumétrica se estimo oportuno aplicar el criterio de intervención "arqueológica", o sea, aquel que incorpora a una obra sus partes faltantes desde un punto de vista lo más neutro posible. El tratamiento de las lagunas reintegradas simulan la textura y el color de la pasta cerámica perdida, se sitúan a bajo nivel respecto el esmalte cerámico y no se ha aplicado ningún elemento gráfico, ni cromático. [Figura 7].

Es evidente que nos encontramos ante una obra con un alto nivel de pérdidas, próximo a lo que podríamos considerar como una ruina y con una considerable merma de su significado simbólico, así como transmisión literaria de sus varias inscripciones. Por ello, se decidió presentar la obra original con esta tipología de reintegración neutra para su exposición museística y estudiar una alternativa, a partir de una reintegración virtual (Aparicio y Figueiredo 2017). Esta propuesta de reconstrucción hipotética que se pretende exponer conjuntamente con el original, permitirá al público comprender y aproximarse con mayor facilidad al sentido primigenio de la obra (Coll y Sanz 2001).

La decisión de aplicar a la obra original un tratamiento neutro persigue la intención de enfatizar los restos extraídos en la excavación arqueológica, más allá de las posibles reintegraciones cromáticas aplicables. Además, dadas el gran número de pérdidas y la dificultad de su reintegración completa, estimamos realizar este trabajo de restitución hipotética, no sobre el original, sino a partir de una reconstrucción virtual que podría ser utilizada de diferentes maneras sin alterar, modificar o influir sobre los vestigios rescatados de la excavación. [Figura 8].

El procedimiento técnico seguido para la propuesta de reconstrucción virtual se basó en tratamiento digital de 


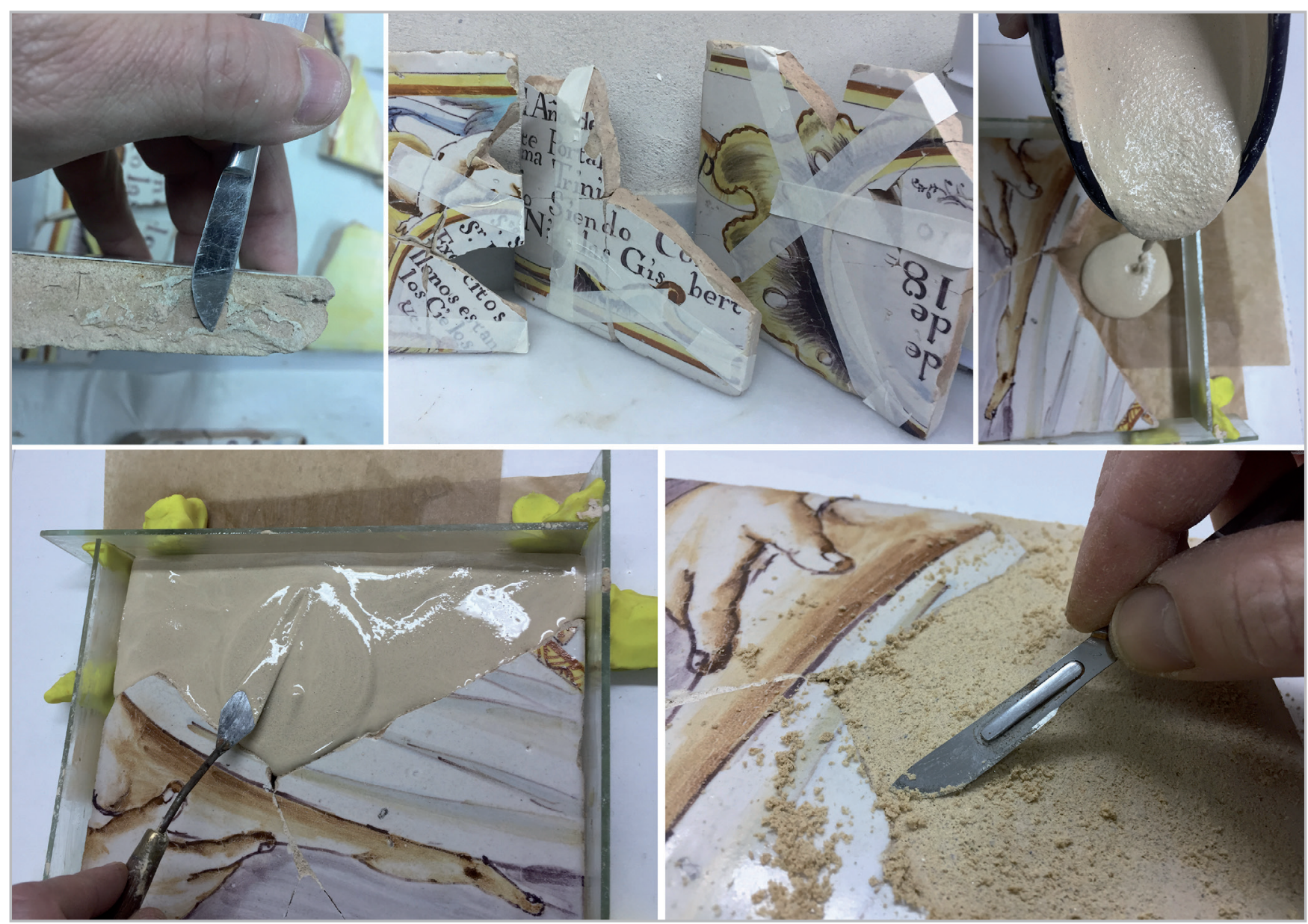

Figura 7.- Secuencia de los procesos de restauración realizados a los azulejos.

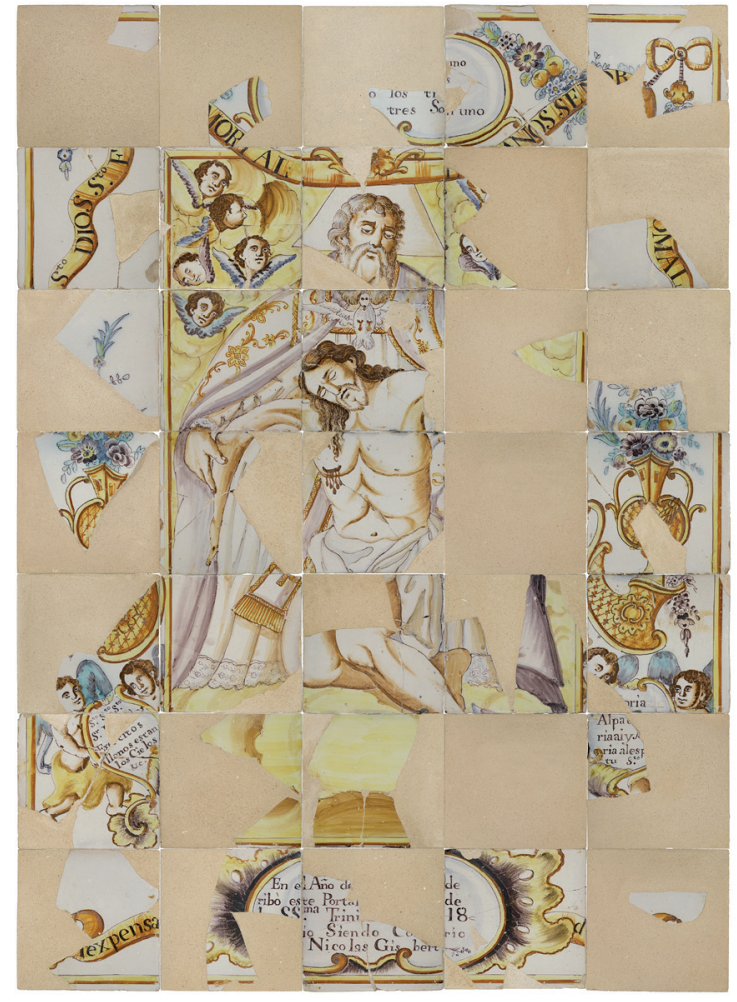

Figura 8.- Composición completa del panel de La Trinidad tras la reintegración volumétrica de los faltantes, bajo un concepto de reintegración neutra. la imagen original, utilizando métodos similares a los que podemos encontrar en otras investigaciones y propuestas precedentes, de donde de se han asimilado algunos parámetros de intervención (Carrascosa y Lastras 2006, Regidor et al. 2006; González y Martínez 2009; Lastras et al .2012; Calero et al. 2020). En primer lugar, todos los azulejos fueron digitalizados con una alta definición con el fin de trabajar las reintegraciones de manera individual en cada unidad que componen el conjunto.

La reintegración digital se ha realizado mediante la utilización del programa detratamiento deimágenesAdobe Photoshop@ en la versión 21.2.0. Procedimentalmente, la conformación de cromatismos y formas sobre las partes perdidas se ha basado en la siguiente metodología técnica:

1.- En cada una de las unidades de reintegración, entendamos esto por cada uno de los azulejos con pérdidas a recuperar, se ha generado una capa independiente delimitada por los extremos de la cerámica y los límites del esmalte original conservado.

2.- Una vez establecida la capa de reintegración, esta era repetida tantas veces como fuera necesario para poder conseguir efectos de capas o distinguir diferentes estratos cromáticos y formales en función de la complejidad de cada zona a reintegrar. 
3.- En este sentido, en primer lugar, se construía una capa inicial con un cromatismo básico de la laguna, en el que se incluía el color y textura del esmalte cerámico y algunas zonas manchadas con el color de fondo.

4.- Posteriormente, en sucesivas capas, todas ellas con la misma configuración formal que la capa de reintegración, se reconstruyen los elementos perdidos: formas, trazos y texturas, los cuales se generaban utilizando herramientas como el "tampón clonar" o copiando y pegando las zonas que por coincidencia cromática y forma pueden corresponder en los espacios perdidos. Las referencias miméticas para conseguir identificar el color y la forma a reconstruir se recuperan, tanto de los entornos cromáticos originales del mismo azulejo, como incluso de otras partes del panel, donde pudiera existir el referente que debía ser incorporado.

Evidentemente, este procedimiento técnico era llevado a cabo sobre cada pérdida tras haber realizado un estudio pormenorizado de los referentes originales existentes. El equilibrio entre zonas perdidas y partes existentes originales, junto la sistematización de los trabajos de estarcido nos han permitido identificar, prácticamente por completo, la gran mayoría de las partes faltantes. La simetría presente en todo el perímetro ornamental del panel y la repetición figurativa de otros elementos como los querubines han facilitado la recuperación hipotética de las partes con mayor carga narrativa y formal. [Figura 9]
No obstante, también se presentan lagunas en zonas comprometidas con faltas de referentes directos que han dificultado la interpretación y ajuste de la propuesta. En estos casos, han sido las fuentes gráficas las que nos han permitido hacer esta aproximación a su reformulación de la manera más referenciada posible. Este ejemplo se ha de manifestar para la reintegración del pie izquierdo de la figura de Jesucristo, el cual coincidía en una zona en la que el azulejo desaparecido.

En este caso, hemos podido identificar fuentes indirectas a través de las cuales basar nuestra hipótesis de reconstrucción, a partir del lienzo de Luis Tristán (Toledo, 1586-1624) de 1624 o el grabado que Jacob Frey (Lucerna, 1681-1752) que en 1734 realiza de la obra pintada por Guido Reni (Bolonia, 1575-1642) sobre este mismo tema. Con estos elementos podemos plantear hipotéticamente cual sería la composición de esta zona faltante de la anatomía de Cristo y, conjuntamente, utilizando los cromatismos de las carnaciones existentes en las partes de esmalte conservadas, atender la reconstrucción de parte de la anatomía.

Otro de los problemas de reintegración de esta obra ha sido la perdida de fragmentos con inscripciones y textos. En este sentido, la búsqueda de documentación bibliográfica y de archivo ha resultado fundamental para superar esta dificultad. Gracias al trabajo y localización de los acontecimientos históricos que rememora este panel devocional. Fundamental ha sido la labor por parte de archiveros y arqueólogos (Belda
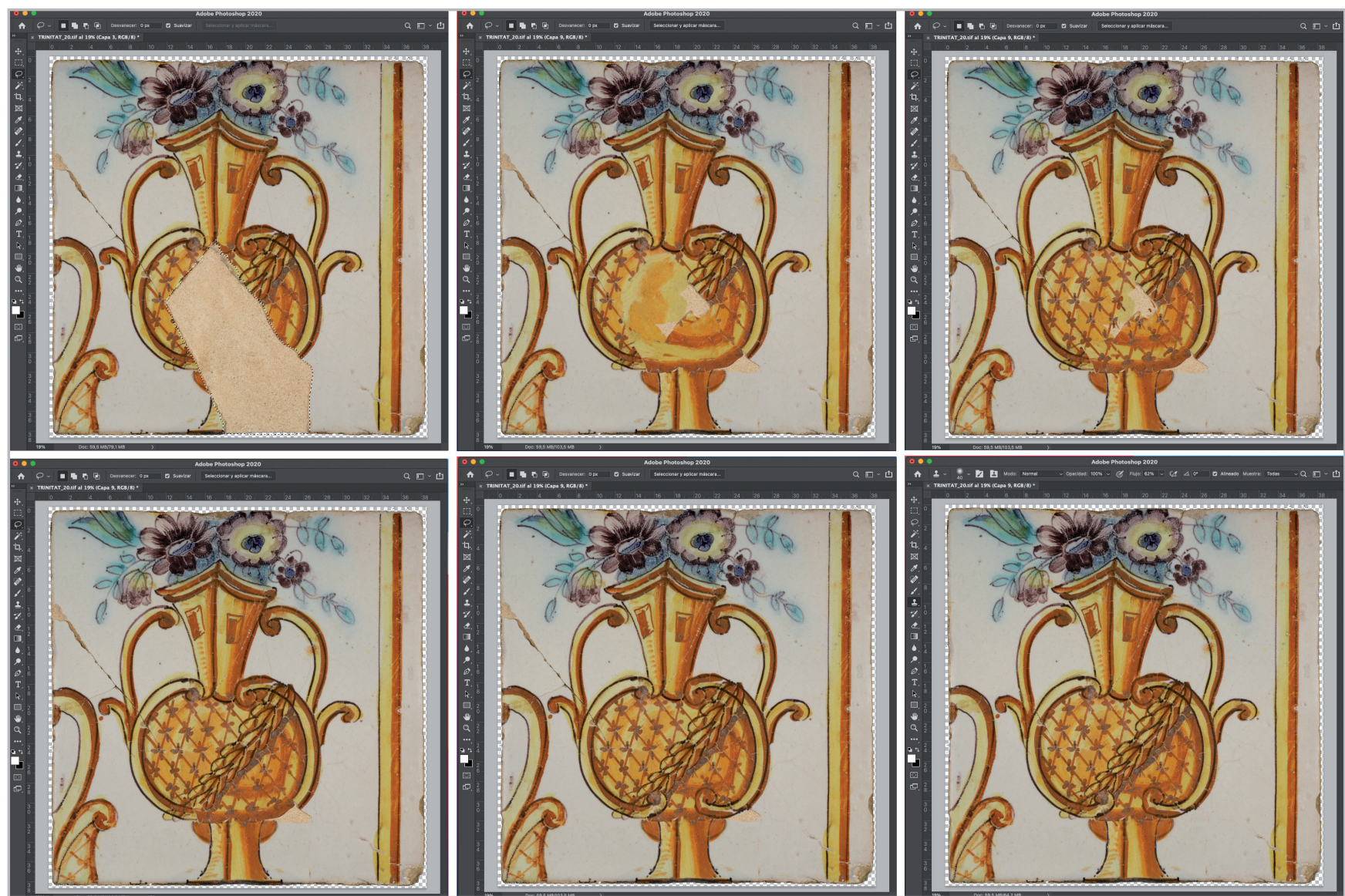

Figura 9.- Secuencia del proceso de reintegración virtual de uno de los azulejos. 
2001; Ribera y Belda 2003) que han permitido determinar algunos datos históricos referidos en el cartucho inferior. Los textos referidos a versículos del Nuevo Testamento se han podido identificar a partir de las letras preservadas. Siendo relativamente viable la reconstrucción de los textos de los cartuchos laterales, y suponiendo un mayor reto aquella que encabeza el panel y que deriva, sin duda, de la "coma joánica" a partir de los versículos de la Primera Epístola de San Juan 5:7-8. [Figura 10]

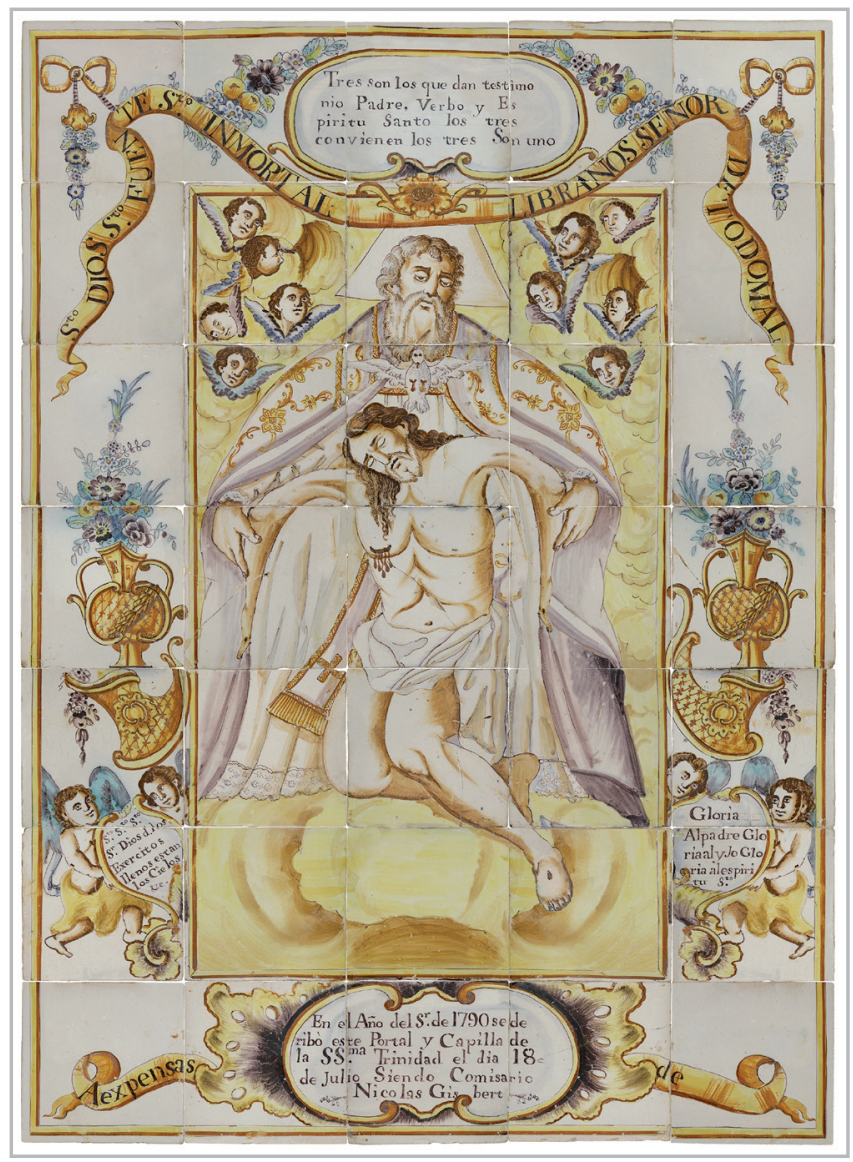

Figura 10.- La Trinidad con la reintegración hipotética virtual.

\section{Conclusiones}

Con nuestro trabajo hemos tratado de ordenar y exponer las soluciones que en materia de reintegración formal y cromática se están llevado a cabo en la actualidad en materia de azulejería, desde nuestra experiencia en el entorno de la azulejería valenciana de los siglos XVIII y XIX. De entrada, cabría adoptar dos posiciones diferentes de intervención, bien se trate de cerámica ornamental arquitectónica, que repite un modelo estandarizado, o de cerámica narrativa de paneles devocionales y costumbristas que requiere de una investigación y búsqueda de fuentes gráficas para tratar de reintegrar partes faltantes de su consistencia formal.

Con nuestro discurso hemos tratado de ordenar las fórmulas de intervención desde las posiciones más conservacionistas y menos intervencionistas, hasta los supuestos de realización de ex novos. Además, hemos tratado de ejemplificar a través del panel de La Trinidad de Ontinyent una cuestión que va más allá de la intervención en este caso neutra en la obra para adentrarnos en el terreno de la museografía y proponer una intervención virtual que permita a los espectadores una comprensión los más próxima posible a lo que fue la obra en su origen, sin menoscabo de la intervención en el original.

\section{Referencias}

APARICIO RESCO, A., \& FIGUEIREDO, C. (2017).“El grado de evidencia histórico-arqueológica de las reconstrucciones virtuales: hacia una escala de representación gráfica". Revista Otarq: Otras arqueologías, 1: 235-247. https://doi.org/10.23914/otarq.v0i1.96

BELDA, J. M. (2001). "L’antic plafó ceràmic de la Trinitat. Ontinyent" Almaig, estudis i documents, 17: 29-33

BALDINI, U. (1981). Teoria del restauro e unitá di metodología. (volúmen 2). Florencia. Nardini Editore.

BERGUEON, S. (1990). Science et patience au la restauration des peintures. París. Réunions des musées Nationaux.

BRANDI, C. (2002). Teoría de la restauración. Madrid. Alianza Forma.

CALERO-CASTILLO, A. I., CARRASCO-HUERTAS, A., DURBÁNGARCÍA, M., DURÁN-SUÁREZ, J. A. (2020). “Documentación y reconstrucción virtual en restauración de obras pictóricas de gran formato: el lienzo mural de la farmacia Zambrano". Virtual Archaeology Review, 11(23): 141-152. https://doi.org/10.4995/ var.2020.13343

CARRASCOSA MOLINER, B \& LASTRAS PÉREZ, M. (2006). La conservación y restauración de la azulejería. Universitat Politècnica de València.

CASAZZA, O. (1981). II restauro pittorico nell'unitá di metodología. Florencia. Nardini Editore.

COLL CONESA, J.; \& SANZ NÁJERA, M. (2001). El azulejo en el museo. Su conservación y montaje expositivo. Museo Nacional de Cerámica y de las Artes Suntuarias González Martí. Madrid. Ministerio de Cultura y Deporte.

GIRONÉS SARRIÓ, I. \& GUEROLA BLAY, V. (2008). “El plafó ceràmic de la Mare de Déu d'Agres del carrer de Cantereria d'Ontinyent: la recuperació d'un patrimoni amagat". Revista Almaig, estudis $i$ documents, 26: 35-39.

GIRONÉS SARRIÓ, I. \& GUEROLA BLAY, V. (2014) "Exemples of tile Works reintegration systems based studyng their graphic sources". Oporto (Portugal). II International Meeting on Retouching of Cultural Heritege (RECH2).

GIRONÉS SARRIÓ, I. \& GUEROLA BLAY, V. (2016). La taulelleria valenciana dels segles XVII, XVIII i XIX a la col-lecció de la Fundació La Fontana. València: Institució Alfons el Magnànim. Centre valencià d'estudis i d'investigació. 
GONZÁLEZ LÓPEZ, M. J., \& MARTíNEZ CALVO, V. (2009). "Reintegración virtual de lagunas. Las pinturas de la Sala de los Reyes de la Alhambra de Granada". Revista pH, 70: 98-113. https:// doi.org/10.33349/2009.70.2754

GUEROLA BLAY, V. (2003). La pintura ceràmica a Carcaixent. Estudi, classificació i catàleg raonat. València: Universitat Politècnica de València - Ajuntament de Carcaixent.

GUEROLA BLAY, V. (2008). "Ideología y metodología en la reintegración de lagunas". II pensiero di Cesare Brandi delle teoría a le practice. Valencia-Roma. Universitat Politècnica de València. Associazione Giovanni Secco Suardo. 110-114.

GUEROLA BLAY, V. (2010). “Pérdidas y perjuicios, reintegraciones y prejuicios". 1er Congreso internacional de restauración de pintura sobre lienzo de gran formato. Valencia. Instituto Universitario de Restauración del Patrimonio, UPV. 451-466.

LASTRAS PÉREZ, M.; MARTÍNEZ BAZÁN, M. L.; MARTÍNEZ CABEZUELO, E. (2012). "Estudio de reintegrantes pictóricos aplicados en la restauración de azulejería expuesta al exterior". Arché, 6: 221-228. http://hdl.handle.net/10251/33515

REGIDOR ROS, J. L., PALUMBO, M., GÓMEZ CHAPARRO, G. \& CLAVEL PIÁ, I. (2006). “Restauración y solución propuesta para la exposición de los fragmentos conservados en la bóveda de la iglesia de los Santos Juanes de Valencia". Arché, 1: 45-52. http://hdl.handle. net/10251/32363

RIBERA, A., BELDA, J. M. (2003). “El Portal i la Capella de la Trinitat: seguiment arqueològic de les obres de rehabilitació en les cases núm. 4 i 6 del carrer Trinitat. La Vila. Ontinyent". 2000. Almaig, estudis i documents, 19: 16-24

RIBERA, A. (2006). "Museu Arqueològic d'Ontinyent i la Vall d'Albaida. Actuació de l'any 2000. Treballs i resums de dades". Alba: revista d'estudis comarcals de la Vall d'Albaida, 20-21: 218-242

\section{Autor/es}

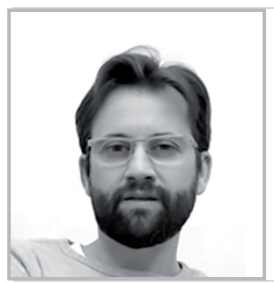

Ignasi Gironés Sarrió
igirones@irp.upv.es
Instituto Universitario de Restauración
del Patrimonio. Universitat Politècnica de
València
https://orcid.org/0000-0003-0540-3880

Doctor en Bellas Artes por la Universitat Politècnica de València, actualmente es Técnico Superior de Apoyo a la Investigación del Instituto Universitario de Restauración del Patrimonio de la UPV. Formado como restaurador de obras de arte, a lo largo de su carrera ha trabajado en centros de restauración de Alemania i Italia, i ha desarrollado una amplia serie de proyectos, tanto de restauración de bienes patrimoniales como de gestión cultural. Su investigación científica se centra en la conservación y restauración de pinturas y obras cerámicas, la cual se refleja en numerosas publicaciones y participaciones en congresos internacionales.

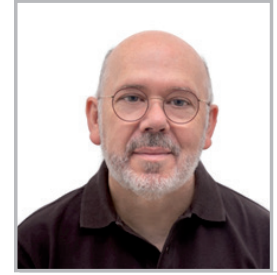

Vicente Guerola Blay
vguerola@crbc.upv.es
Instituto Universitario de Restauración
del Patrimonio. Universitat Politècnica de
València
https://orcid.org/0000-0001-5223-7737

Licenciado en Bellas Artes por la Facultad de Bellas Artes de San Carlos de Valencia y doctor por la Universidad Politécnica de Valencia dondeactualmenteejercesulabor docentecomo profesor titular en el Departamento de Conservación y Restauración de Bienes Culturales. Es especialista en pintura cerámica valenciana, labor a la que ha dedicado diferentes artículos y monografías. Ha publicado diversas monografías en materia de restauración de pintura destacando su especial dedicación en materia de retoque y criterios de reintegración pictórica. En esta misma disciplina ha participado en diferentes foros, congresos y jornadas aportando a través de artículos y ponencias diversas casuísticas y procesos de intervención en el patrimonio histórico-artístico. Ha participado como Jefe de taller y director en la restauración de diferentes conjuntos monumentales destacando su labor al frente de la restauración de la colección pictórica de la Basílica de Ntra. Sra. de los Desamparados de la ciudad de Valencia, en el conjunto de la pinturas de la Galería Dorada del Palacio ducal de Gandía o en la colección de pintura sobre lienzo y tabla de la histórica parroquia de San Nicolás, también en la ciudad de Valencia. Desde hace unos años participa a través de diferentes proyectos de investigación en el estudio de la Tomografía Axial Computerizada aplicada al análisis de obras de arte, con una destacada aportación de publicaciones en esta materia. Ha dirigido un proyecto de investigación europeo en materia de estudio del Patrimonio Efímero concedido dentro del programa Europa Creativa (Ephimeral Heritage in european carnival rituals) y se encuentra al frente como Conservador de la Colección pictórica de la Fundación Colegio Asilo de Ntra. Sra. de los Desamparados de Carcaixent.
Artículo enviado el 23/09/2021 Artículo aceptado el 15/10/2021 (c)

https://doi.org/10.37558/gec.v20i1.1054 